\title{
Ensino interativo na abordagem da eletricidade numa escola portuguesa ${ }^{+*}$
}

\section{J. Quintas ${ }^{1}$}

Agrupamento de Escolas de Valbom

Gondomar - Portugal

Instituto de Física dos Materiais

Universidade do Porto

P. Simeão Carvalho ${ }^{2}$

Instituto de Física dos Materiais

Universidade do Porto

Porto - Portugal

\section{Resumo}

A exploração de materiais didáticos interativos é vista como potenciadora de uma melhor aprendizagem da Física. Neste trabalho apresentamse alguns resultados obtidos da realização de atividades práticas de laboratório (APL) e exploração de ferramentas baseadas em recursos educativos digitais (RED), na abordagem da Eletricidade em algumas escolas portuguesas. Nesta investigação, os alunos foram divididos em Grupo Experimental (GE) e Grupo de Controlo (GC). Na recolha dos dados foram usadas fichas, relatórios de aula, inquéritos e entrevistas. Os resultados apontam para uma melhor aprendizagem dos alunos do GE, validando assim as hipóteses de qualidade e de continuidade desta prática de ensino e aprendizagem nas escolas. Este trabalho mostra também que quer a utilização pelos alunos dos materiais interativos quer a formação dos professores na exploração desses materiais, são necessárias para promover uma aprendizagem colaborativa.

\footnotetext{
${ }^{+}$Interactive education in the approach of electricity in a portuguese school

* Recebido: novembro de 2015. Aceito: junho de 2016.

${ }^{1}$ E-mail: quintas.mariajose@gmail.com

2 E-mail: psimeao@fc.up.pt
} 
Palavras-chave: Ensino interativo; Circuitos elétricos; Atividades práticas laboratoriais; Software educativo; Vídeo educativo; WebQuest; Ganhos de aprendizagem.

\begin{abstract}
The use of interactive teaching materials is seen as a booster to improve Physics learning. In this paper, we present some results obtained while performing laboratorial practice activities (LPA) and exploring tools based on digital educational resources (DER), while teaching electricity in some portuguese schools. In this investigation, students were divided into Experimental Group (EG) and Control Group (CG). Forms, class reports, surveys and interviews were used to collect data. The results point to a better learning by the EG students, thus validating the hypotheses of quality and continuity of this practice of teaching and learning in schools. This work also shows that both the use of interactive materials by the students and in teacher training are necessary to promote collaborative learning.
\end{abstract}

Keywords: Interactive teaching; Electric circuits; Laboratory Practical Activities; Educational software; Educational video; WebQuest; Learning gains.

\title{
I. Introdução
}

Nos últimos vinte anos um número crescente de físicos tem contribuído ativamente para o avanço do ensino da Física em todos os níveis escolares, com propostas e investigações didáticas publicadas em livros (MAZUR, 1997; ARONS, 1997; SOKOLOFF; THOMTON; LAWS, 1999, 2004; MCDERMOTT, 1995, 1996; REDISH, 2003; KNIGHT, 2004) e em artigos científicos (HAKE, 1998; HOFSTEIN; LUNETTA, 2004). Na sua globalidade, os resultados destas investigações indicam que há um desfasamento acentuado entre o ensino de conteúdos nas salas de aula e a aprendizagem efetiva desses conteúdos pelos alunos.

Em Portugal, o panorama descrito não é diferente. Lopes e Silva (2009) apontam que nas escolas Portuguesas, ainda se privilegia o individualismo e a competição entre os alunos. $\mathrm{Na}$ dinamização dos trabalhos de grupo, alguns professores oferecem resistência em abandonar as metodologias tradicionais, não se verificando um efetivo espírito de cooperação e aprendizagem em grupo.

Esta realidade conduz a novos desafios para as escolas e para os professores em particular, a fim de manter os alunos mentalmente ativos nas salas de aula e de promover ambientes mais favoráveis para a aprendizagem. 
Optamos por levar em linha de conta a visão de Sokoloff (1997), que defende que a realização de atividades práticas de laboratório (APL) conduz à compreensão e assimilação de conceitos da Física, uma ciência reconhecidamente experimental. Esta visão do ensino e aprendizagem é tão indispensável como aquelas que têm por base a utilização de recursos educativos digitais (RED) e o uso das Tecnologias de Informação e Comunicação (TIC) (NOVAK et al., 1999). Na sua globalidade, as APL, os RED e em geral as TIC, disponibilizam ao professor um leque de opções letivas que irão certamente ao encontro da panóplia de metodologias existentes, conducentes à eficaz aprendizagem conceitual pelos estudantes. Será, assim, necessário disponibilizar vários recursos materiais e tecnológicos ao professor e dar-lhe a formação necessária, para que ele tenha a capacidade de os utilizar. Desta forma, o professor poderá criar um ambiente de debate, de confronto de ideias e de análise crítica de situações, que favoreçam um ambiente propício para os estudantes tirarem conclusões cientificamente corretas sobre os conteúdos lecionados.

Neste trabalho, mostramos como a conjugação de APL e RED pode afetar positivamente a aprendizagem em eletricidade de estudantes de algumas escolas portuguesas. Também se discute como a formação de professores na exploração de recursos didáticos interativos pode ter um efeito significativo na aprendizagem dos estudantes.

\section{Caracterização da investigação}

A investigação foi realizada ao longo de dois anos letivos, 2013/ 2014 e 2014/ 2015, em três escolas públicas portuguesas do distrito de Lisboa, no $9 .^{\circ}$ ano do ensino básico português.

Nela, foram elaborados e usados vários recursos educativos interativos alusivos a conteúdos programáticos sobre Eletricidade: 11 Atividades Práticas de Laboratório (APL); 8 Roteiros de Exploração de Software Educativo (RESE); 11 Roteiros de Exploração de Vídeo Educativo (REVE); 1 WebQuest; 9 Quizzes. A estrutura e tipologia de cada um destes recursos é apresentada no Anexo A, como exemplo.

\section{Ano letivo 2013/2014}

No primeiro ano do estudo (2013/2014), estiveram envolvidos 108 alunos e 3 professoras pertencentes à Escola EB 2,3 Roque Gameiro - Amadora (distrito de Lisboa).

Neste estudo, pretendeu-se compreender qual o impacto da formação dos professores na aprendizagem dos alunos. Assim, todas as professoras titulares das turmas (e respectivos alunos) tiveram acesso às APL e aos RED; a diferença entre o Grupo de Controlo (GC) e o grupo experimental (GE) foi ao nível da formação dos professores na exploração didática dos recursos educativos:

GE - foi prestado auxílio às professoras na implementação dos recursos;

GC - as professoras não tiveram qualquer formação na exploração dos recursos. 


\section{Ano letivo 2014/2015}

No segundo ano letivo (2014/2015), estiveram envolvidos 160 alunos e 5 professores. A intervenção foi feita em duas escolas pertencentes ao distrito de Lisboa e concelho de Cascais.

Neste estudo pretendeu-se compreender qual o impacto na aprendizagem dos alunos, da conjugação dos recursos educativos interativos com a formação especializada em metodologia interativa na prática letiva. Assim, na Escola Básica de Alcabideche (grupo experimental, GE), os recursos educativos foram explorados por um dos autores, recorrendo a metodologias ativas como a Peer Instruction (Instrução pelos colegas - IpC) (MAZUR, 1997), cujo foco principal se baseia no questionamento e o Just-in-Time Teaching (Ensino sob Medida EsM) (NOVAK et al., 1999), que tem como linha orientadora na arquitetura de cada aula o conhecimento prévio dos alunos. Por oposição, na Escola Secundária IBN-Mucana (Grupo de Controlo, GC) os alunos tiveram um ensino tradicional (metodologia expositiva) e sem acesso a recursos educativos interativos.

Cabe salientar que no Ranking de 2013 das escolas participantes neste estudo (ordenação das escolas portuguesas do ensino básico, tomando a média das classificações dos exames nacionais de Português e Matemática), a escola do GE ocupava uma posição inferior (1096 lugares abaixo) à da escola do GC.

\section{Avaliação}

Como instrumentos de avaliação usou-se: (i) Ficha de Caracterização; (ii) Teste diagnóstico; (iii) Pré-Teste de conhecimentos; (iv) Pós-Teste de conhecimentos; (v) Inquérito, (vi) Entrevistas.

A tabela 1 resume os instrumentos aplicados em cada grupo de estudo (GE e GC), nos anos letivos correspondentes.

Tabela 1 - Instrumentos de avaliação aplicados neste estudo.

\begin{tabular}{|l|c|c|c|c|}
\hline \multirow{2}{*}{\multicolumn{1}{|c|}{ Instrumentos }} & \multicolumn{2}{|c|}{2013 2014 } & \multicolumn{2}{c|}{2014 2015 } \\
\cline { 2 - 5 } & $\begin{array}{c}\text { ALUNOS } \\
\text { (GE/ GC) }\end{array}$ & Professoras & $\begin{array}{c}\text { ALUNOS } \\
\text { (GE) }\end{array}$ & $\begin{array}{c}\text { ALUNOS } \\
\text { (GC) }\end{array}$ \\
\hline $\begin{array}{l}\text { - Ficha de Caracterização do(a) } \\
\text { aluno(a) }\end{array}$ & $\mathrm{X}$ & $\ldots$ & $\mathrm{X}$ & $\mathrm{X}$ \\
\hline - Teste Diagnóstico & $\mathrm{X}$ & $\ldots$ & $\ldots$ & $\ldots$ \\
\hline - Pré - Teste de conhecimentos & $\mathrm{X}$ & $\ldots$ & $\mathrm{X}$ & $\mathrm{X}$ \\
\hline - Pós-Teste de conhecimentos & $\mathrm{X}$ & $\ldots$ & $\mathrm{X}$ & $\mathrm{X}$ \\
\hline - Inquérito & $\mathrm{X}$ & $\ldots$ & $\mathrm{X}$ & $\ldots$ \\
\hline - Entrevista áudio & $\mathrm{X}$ & $\mathrm{X}$ & $\mathrm{X}$ & $\ldots$ \\
\hline
\end{tabular}


O teste diagnóstico é constituído por 10 questões de resposta fechada e tem como objetivo apurar as concepções prévias dos alunos. O teste avalia a compreensão dos alunos em tópicos diversos, tais como eletricidade estática, montagem de circuitos elétricos, analogia hidráulica com a passagem da corrente eléctrica, caracterização dos componentes elétricos e mecanismos de passagem de corrente elétrica, caracterização dos circuitos em série e distinção entre corrente elétrica e diferença de potencial. Com a informação recolhida neste teste, planificaram-se estratégias de ensino adequadas à aprendizagem em grupo da eletricidade e à promoção do Ensino sob Medida - EsM (NOVAK et al., 1999).

O teste de conhecimentos é constituído por 23 questões de escolha múltipla, construído com base no trabalho de Engelhardta e Beichner (2004) e no teste conceitual de Thornton e Sokoloff (2005). O teste avaliava os conhecimentos de corrente eléctrica / circuitos elétricos e os efeitos da corrente eléctrica. Foi aplicado antes e após a exploração da eletricidade (pré e pós-teste), com o intuito de avaliar ganhos de aprendizagem. Algumas das questões do teste encontram-se disponíveis no Anexo B.

O inquérito foi dado no final do ano letivo e contempla questões de resposta aberta/ fechada. Está subdividido em quatro secções. Na primeira, questiona-se a opinião dos alunos quanto: (i) à qualidade dos recursos e seu enquadramento no âmbito das temáticas da Ciência, Tecnologia, Sociedade e Ambiente (CTSA); (ii) ao interesse para que estas estratégias interativas sejam aplicadas em anos subsequentes; (iii) ao contributo das metodologias usadas nas aulas para um melhor rendimento/ desempenho escolar. Na segunda e terceira secções, os alunos puderam expressar em que medida a realização de APLs e a exploração dos REDs contribuíram para uma melhor aprendizagem dos conceitos. Por fim, na última secção foi solicitado aos alunos que avaliassem as estratégias de ensino e aprendizagem aplicadas e indicassem sugestões.

\section{Resultados}

Os dados recolhidos com a aplicação dos instrumentos de avaliação foram alvo de análise qualitativa e quantitativa, seguindo uma metodologia quasi-experimental semelhante à proposta por Campbell e Stanley (1979).

No teste diagnóstico aplicado no ano letivo 2013/2014, os resultados evidenciaram que os alunos possuíam a maioria das concepções prévias apontadas na literatura. Em geral, as concepções prévias estão fortemente enraizadas na experiência diária dos alunos e uma das formas de promover a mudança conceitual é através do debate de ideias (ARONS, 1997). Assim, este resultado veio confirmar que estavam reunidas condições educativas para o estudo de impacto de uma prática de ensino e aprendizagem interativa.

Ao nível do conhecimento dos alunos no início de cada ano letivo, não se registraram diferenças significativas entre os vários grupos de estudo. Tal constatação significa que, de partida, o grau de conhecimento dos alunos em cada ano letivo era o mesmo.

No final de cada ano letivo fez-se uma análise aos ganhos de aprendizagem de todos 
os grupos de alunos. Foram então calculados:

(i) Ganho absoluto médio: $\mathrm{G}_{\mathrm{a}}=\mathrm{N}_{\mathrm{f}}-\mathrm{N}_{\mathrm{i}}$

no qual $\mathrm{N}_{\mathrm{i}}$ é a classificação média do pré-teste e $\mathrm{N}_{\mathrm{f}}$ é a classificação média do pós-teste.

(ii) Ganho relativo médio $\left(\mathrm{G}_{\mathrm{r}}\right)$, dado por Hake (1998):

$$
G_{r}=\frac{N_{f}-N_{i}}{100-N_{i}} \times 100 \%
$$

A tabela 2 resume, por ano letivo, os grupos experimentais e de controlo, a classificação média percentual no pré e pós-teste de conhecimentos $\left(\mathrm{N}_{\mathrm{i}}\right.$ e $\mathrm{N}_{\mathrm{f}}$, respectivamente), o ganho absoluto médio $\left(\mathrm{G}_{\mathrm{a}}\right)$ e o ganho relativo médio $\left(\mathrm{G}_{\mathrm{r}}\right)$, este último refletindo melhor o grau de aprendizagem do aluno comparativamente ao seu nível inicial de conhecimento.

Tabela 2 - Resumo dos resultados obtidos em todos os grupos de estudo.

\begin{tabular}{cc|cccc}
\hline Ano letivo & $\begin{array}{c}\text { Grupo de } \\
\text { intervenção }\end{array}$ & $\mathbf{N}_{\mathrm{i}}(\boldsymbol{\%})$ & $\mathbf{N}_{\mathrm{f}}(\boldsymbol{\%})$ & $\mathbf{G}_{\mathrm{a}}(\boldsymbol{\%})$ & $\mathbf{G}_{\mathrm{r}}(\%)$ \\
\hline \multirow{2}{*}{$\mathbf{2 0 1 3 / 2 0 1 4}$} & GE & $\mathbf{3 2 , 3 0}$ & $\mathbf{4 3 , 1 3}$ & $\mathbf{1 0 , 8 3}$ & $\mathbf{1 5 , 9 9}$ \\
& GC & 32,50 & 35,84 & 3,33 & 4,91 \\
\hline \multirow{2}{*}{$\mathbf{2 0 1 4 / 2 0 1 5}$} & GE & $\mathbf{2 3 , 4 5}$ & $\mathbf{5 4 , 0 4}$ & $\mathbf{3 0 , 5 9}$ & $\mathbf{3 9 , 9 6}$ \\
& GC & 29,87 & 42,36 & 12,48 & 17,80 \\
\hline
\end{tabular}

Em cada ano letivo, os valores do ganho absoluto de aprendizagem $\mathrm{G}_{\mathrm{a}}$ entre o grupo experimental e o Grupo de Controlo são significativamente diferentes. Em todos os casos, os alunos do $\mathrm{GE}$ apresentam um $\mathrm{G}_{\mathrm{a}}$ superior (mais do dobro) comparativamente aos do GC desse mesmo ano letivo.

Os resultados da tabela 2 estão sintetizados graficamente na Fig. 1, onde as linhas vermelha, azul e verde representam os ganhos percentuais relativos de $10 \%, 20 \%$ e $40 \%$, respectivamente.

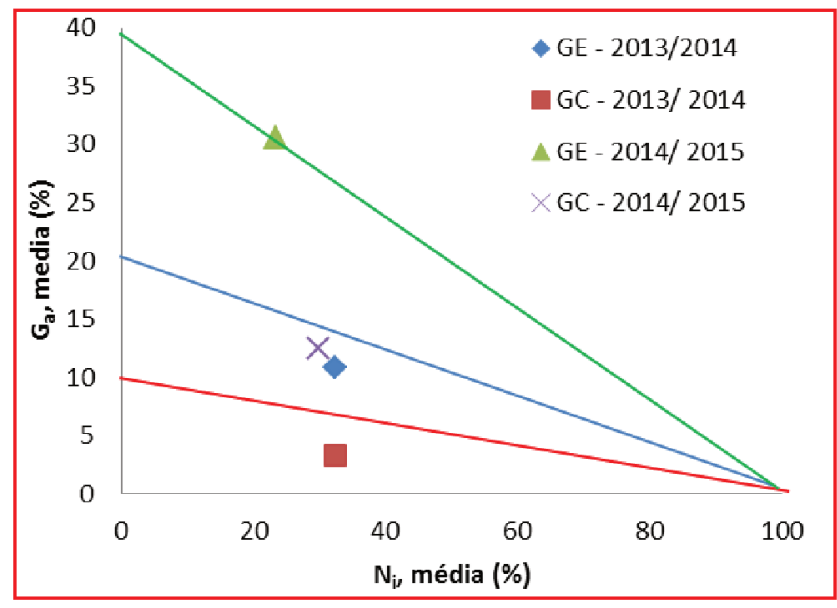

Fig. 1 -Representação do $G_{a}$ em função da $N_{i}$.

No ano letivo de $\mathbf{2 0 1 3 / 2 0 1 4}$, obtiveram-se valores de $\mathrm{G}_{\mathrm{r}}$ muito abaixo do esperado, 
tendo em conta que todos os alunos tiveram acesso a materiais interativos durante a prática letiva. Mesmo assim, as diferenças nos valores registradas nos grupos GE e GC mostram inequivocamente que os recursos interativos não produzem, por si só, ganhos significativos de aprendizagem. Este resultado nem sempre é óbvio para todos os professores, até porque na literatura é frequente encontrarem-se propostas de materiais didáticos (por exemplo, animações ou vídeos educativos) sem sugestões de exploração; este nosso estudo sugere que essa componente condiciona a aprendizagem efetiva dos alunos. Conclui-se então que é necessária uma exploração bem orientada dos recursos interativos para que eles tenham um impacto efetivo na aprendizagem dos estudantes.

No ano letivo de $\mathbf{2 0 1 4 / 2 0 1 5}$, o GC teve um valor de $\mathrm{G}_{\mathrm{r}}$ próximo dos $20 \%$, consistentes com os relativos à aprendizagem com uma metodologia tradicional e tendo como referência outros testes de conhecimento conceitual (MAZUR, 1997; SAVINAINEN; SCOTT, 2002). Contudo, o GE apresenta ganhos relativos de aprendizagem significativamente superiores, próximos dos $40 \%$ (região que pode ser associada à aprendizagem com um ensino interativo).

O elevado aumento de aprendizagem registrado no GE (2014/2015) está bem ilustrado na Fig. 2, que traduz o número de alunos (expresso em percentagem) em função do número de respostas certas obtidas antes e após a intervenção. A figura evidencia que a taxa de sucesso no pós-teste foi bastante superior à alcançada no pré-teste.

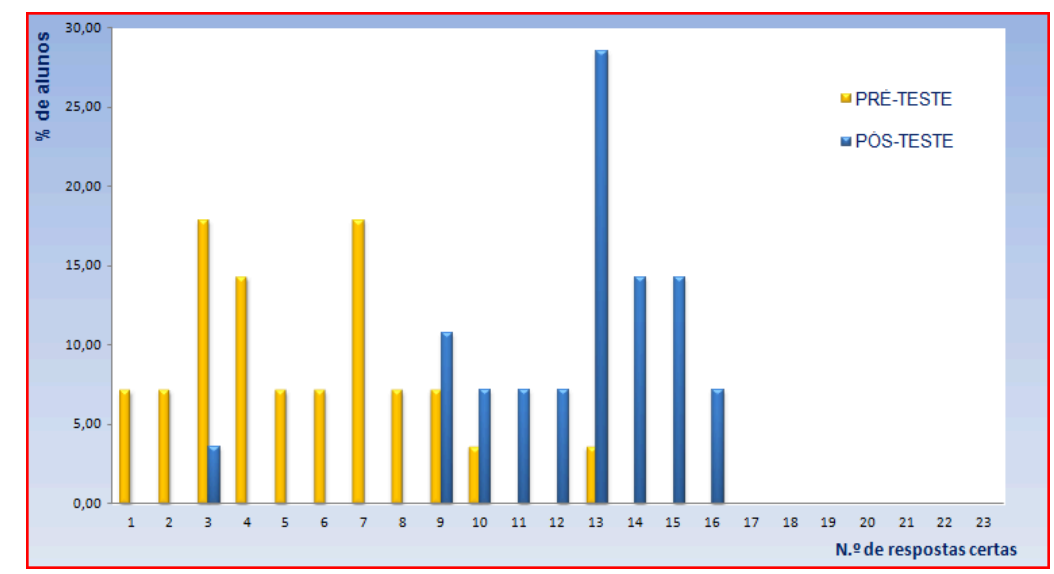

Fig. 2 - Número de alunos (expresso em percentagem) em função do número de respostas certas para o GE (2014/2015), obtido antes e após a intervenção.

As entrevistas realizadas aos alunos do GE são reveladoras do impacto positivo das APLs e dos REDs. A título de exemplo, transcrevemos alguns desses comentários (as frases são transcrições integrais; apenas foram corrigidos erros de ortografia):

- "Senti-me mais motivada, porque logo de início, havia várias estratégias."

- "Acho que estas estratégias me ajudaram bastante, porque eu não gosto muito de estudar pelos livros e acho que estas estratégias me motivaram mais, porque são aplicados 
vídeos, há recursos interativos e assim, favorece muito mais a minha atenção.”

- "Estas estratégias favoreceram a minha aprendizagem porque eu no $1 .^{\circ}$ e $2 .^{\circ}$ períodos [de aulas] tive [nota] negativa a Física e Química e estas estratégias de ensino conseguiram-me cativar mais nas aulas e assim, prestar mais atenção e desenvolver o meu empenho na disciplina."

APL: "Estávamos a fazer as tarefas já sem o livro"; "estávamos a pôr à prova aquilo que aprendíamos"; "podíamos ter mais uma noção da realidade".

RESE: "Simular situações que na realidade podiam correr bem ou mal.”; "É mais interessante mexer no computador, observar coisas que não conseguimos fazê-lo a olho nu e/ou no livro.”; “...podia chegar a casa e voltar a fazer”.

REVE: "A matéria era descrita no vídeo e em pouco tempo (sem recorrer ao livro), podendo aproveitar o tempo da aula para outras atividades".

Quiz: "As escolhas múltiplas podiam ajudar-nos mesmo nas avaliações de outras disciplinas."; "Nós teríamos de estar muito atentos para não nos baralharmos e podermos aprender melhor."; "Mas mesmo que numa fase inicial nos sentíssemos baralhados, tínhamos de recordar o que havíamos dado para trás com as APL, REVE, RESE".

WebQuest: "É sempre muito bom pesquisar e descobrir coisas... principalmente em grupo, que é muito mais engraçado".

\section{Conclusões}

Os resultados obtidos neste estudo apontam para um domínio significativamente maior dos conteúdos de Eletricidade pelos alunos dos GE, validando a hipótese do benefício da integração eficaz de ferramentas que promovem um ensino interativo (APL e RED) nas aulas de Física e da formação adequada dos professores de Física.

De uma forma geral, a diferença na aprendizagem, para cada ano letivo, dos grupos GE e GC é relevante e evidencia a influência de vários fatores conjugados: (i) formação adequada na prática letiva; (ii) recursos educativos usados; (iii) metodologia de ensino. Os resultados sugerem que a aprendizagem dos alunos é mais favorável quando os professores são adequadamente preparados para práticas letivas que promovem o trabalho cooperativo, com exploração de recursos e estratégias interativas.

Há quase vinte anos, Hake (1998) sugeriu que para haver um significativo ganho de aprendizagem dos alunos, não são suficientes apenas atividades "mãos na massa" (hands-on); impõe-se a necessidade de envolver os estudantes em tarefas que promovam o uso do "raciocínio" (heads-on), ou seja, para além de manipular os materiais didáticos e laboratoriais, é fulcral estimular o pensamento crítico através de uma intervenção assertiva do professor. Conclusão semelhante é também transmitida pelos trabalhos de Hofstein e Lunetta (1982, 
2004). Os resultados obtidos neste trabalho vêm sugerir, embora para um estudo local, que os problemas de aprendizagem podem ser reduzidos através da preparação do professor em práticas letivas, que potenciam uma postura ativa do aluno e sua participação colaborativa. Estas conclusões, embora possam parecer triviais, na verdade são ainda pertinentes pois persiste ainda uma prática docente muito disseminada, que aprova uma abordagem tradicional dos conteúdos de Física.

Não podemos deixar de referir que a aplicação de metodologias interativas requer um esforço inicial por parte dos professores, tanto no domínio destas ferramentas, como na necessidade de dispor mais do seu tempo na preparação prévia das aulas. Assim, a formação de professores desempenha um papel muito importante no futuro do ensino da Física, nomeadamente otimizando a exploração de materiais inovadores, promovendo a partilha de ideias entre professores e tornando o trabalho de preparação de aulas uma tarefa mais facilitada.

Apesar do esforço e tempo requerido para a execução das atividades neste estudo, a opinião das professoras que participaram nos GE foi unânime, ao afirmarem que tempo gasto "hoje" será rentabilizado "amanhã".

\section{Agradecimentos}

Este trabalho foi financiado pela Fundação para a Ciência e a Tecnologia (FCT), Projeto UID/NAN/50024/2013.

\section{Referências bibliográficas}

ARONS, A.B. Teaching Introductory Physics. New York: John Wiley \& Sons Inc, 1997. $816 \mathrm{p}$.

CAMPBELL, D. T.; STANLEY, J. C. Delineamentos experimentais e quase-experimentais de pesquisa. Tradução: R.A.T. Di Dio. São Paulo: EPU-EDUSP, 1979. 138 p.

ENGELHARDTA, P.; BEICHNER, R. Students' understanding of direct current resistive electrical circuits. American Journal of Physics, Melville, NY, v. 72, n. 1, p. 98-115, jan. 2004.

HAKE, R. R. Interactive-engagement versus traditional methods: A six-thousand-student survey of mechanics test data for introductory physics courses. American Journal of Physics, Melville, NY, v. 66, n. 1, p. 64-74, jan. 1998.

HOFSTEIN, A.; LUNETTA, V. N. The role of the laboratory in science teaching: Neglected aspects of research. Review of Educational Research, New York NY, v. 52, n. 2, p. 201217, 1982.

HOFSTEIN, A.; LUNETTA, V. N. The Laboratory in Science Education: Foundations for the 
Twenty-First Century. Science Education, Malden, v. 88, n. 1, p. 28-54, jan. 2004.

KNIGHT, R. D. Five Easy Lessons: Strategies for Successful Physics Teaching. San Francisco: Addison-Wesley Publishing Company Inc., 2004. 330 p.

LOPES, J.; SILVA, H. S. A aprendizagem cooperativa na sala de aula: Um guia prático para o professor. Lisboa: Lidel, 2009. 305 p.

MAZUR, E. Peer Instruction: a User's manual. Prentice Hall Series in Educational Innovation, Prentice Hall Series in Educational Innovation, Upper Saddle River, NJ, 1997. 253 p.

MCDERMOTT, L. Physics by Inquiry: An Introduction to Physics and the Physical Sciences. New York: John Wiley \& Sons Inc., 1995-1996. 823 p. v. 1 - 2.

NOVAK, G. M.; PATTERSON, E. T.; GAVRIN, A.; CHRISTIAN, W. Just-in-TimeTeaching: Blending Active Learning with Web Technology. Prentice Hall, Upper Saddle River, NJ, 1999. 188 p.

REDISH, E. Teaching Physics with the Physics Suite. New Jersey: John Wiley \& Sons, Inc., 2003. 215 p.

SAVINAINEN, A.; SCOTT, P. The Force Concept Inventory: a tool for monitoring student learning. Physics Education, Bristol, v. 37, n. 1, p. 45-52, jan. 2002.

SOKOLOFF, D. R. Using interactive lecture demonstrations. Physics Teacher, Melville, NY, v. 35, n. 6, p. 340-347, jun. 1997.

SOKOLOFF, D.; THORNTON, R.; LAWS, P. Real Time Physics: Active Learning Laboratories. Hoboken, NJ: John Wiley \& Sons Inc., 1999-2004. v. 1 - 4.

THORNTON, R.; SOKOLOFF, D. The electric circuits concept evaluation. Disponível em: $<$ http://www.physics.umd.edu/perg/tools/diags.htm>. Acesso em: 17 jun. 2016. 


\section{Anexo A - Recursos educativos}

\section{APL - Tipos de circuitos elétricos}

APL 8 - TIPOS DE CIRCUITOS ELÉTRICOS

\section{SABIAS QUE?}

E possivel instalar num circuito elétrico mais do que um recetor. A instalação pode efetuar-se de duas formas diferentes: em série (figura 1) e em paralelo (figura 2).

Num circuito com lâmpadas em série, uma é ligada a seguir à outra e só existe um caminho para a passagem da corrente elétrica. Num circuito com lâmpadas em paralelo, cada uma é instalada numa ramificação diferente, existindo assim, mais do que um caminho para a corrente elétrica. Há um ponto, designado nó, onde a corrente do ramo principal se divide pelas suas ramificações e outro nó onde a corrente se junta

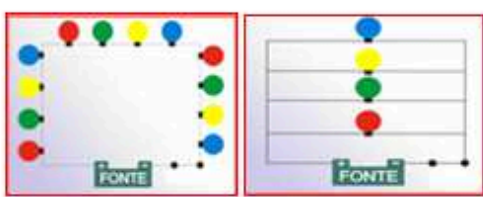

Figura 1 novamente

\section{QUESTÓES MOTIVADORAS}

Como estão instalados os equipamentos elétricos nas nossas casas?

Qual(ais) a(s) vantagem(ns)/ desvantagem(ns) destes dois tipos de associação?

FINALIDADE

$\checkmark$ Construir e interpretar circuitos elétricos com associações de lâmpadas em série e em paralelo. MATERIAL

$\checkmark 1$ pilha de $4,5 \mathrm{~V}$.

$\checkmark$ crocodilos.

$\checkmark 7$ fios de ligação.

$\checkmark 2$ lâmpadas com suporte.

$\checkmark$ Interruptor.

\section{PROCEDIMENTO}

$\checkmark$ Instala o circuito elétrico da figura 3, constituído por uma pilha, lâmpada, interruptor (I) e fios de ligação.

$\Leftrightarrow$ Observa com atenção o brilho emitido pela lâmpada.

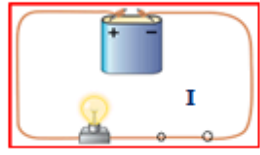

Figura 3

\section{ASSOCIAÇĀO DE LÄMPADAS EM SÉRIE}

$\checkmark$ Instala o circuito elétrico ilustrado na figura 4, colocando 2 lâmpadas em SERIE.

$\Leftrightarrow$ Compara o brilho de cada uma destas lâmpadas (figura 4) com o seu brilho no circuito anterior (figura 3).

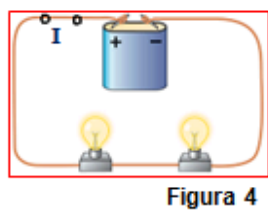

$\Leftrightarrow$ Desenrosca separadamente cada uma das lâmpadas e regista as observações. 


\begin{tabular}{|c|c|c|c|}
\hline $\begin{array}{l}\text { DISCUSSÄO E CONCLI } \\
\text { 1. Completa a seguin }\end{array}$ & täES & & \\
\hline $\begin{array}{l}\text { Circuito elétrico } \\
\text { com... }\end{array}$ & $\begin{array}{c}\text { Esquema do circuito } \\
\text { elétrico }\end{array}$ & $\begin{array}{l}\text { Brilho das lampadas, } \\
\text { quando comparado } \\
\text { com o circuito } \\
\text { anterior (diminuiu/ } \\
\text { aumentou/manteve-se) }\end{array}$ & $\begin{array}{c}\text { O que aconteceu } \\
\text { quando se } \\
\text { desenroscou cada } \\
\text { uma das lâmpadas? }\end{array}$ \\
\hline uma lâmpada & & ................... & ................... \\
\hline $\begin{array}{c}\text {...2 lâmpadas em } \\
\text { SÉRIE }\end{array}$ & & & \\
\hline $\begin{array}{l}\text {...2 lâmpadas em } \\
\text { PARALELO }\end{array}$ & & & \\
\hline
\end{tabular}

2. Explica cientificamente a variação do brilho das lâmpadas, sabendo que este é função da corrente elétrica que a atravessa. 


\section{Rese - Reóstato}

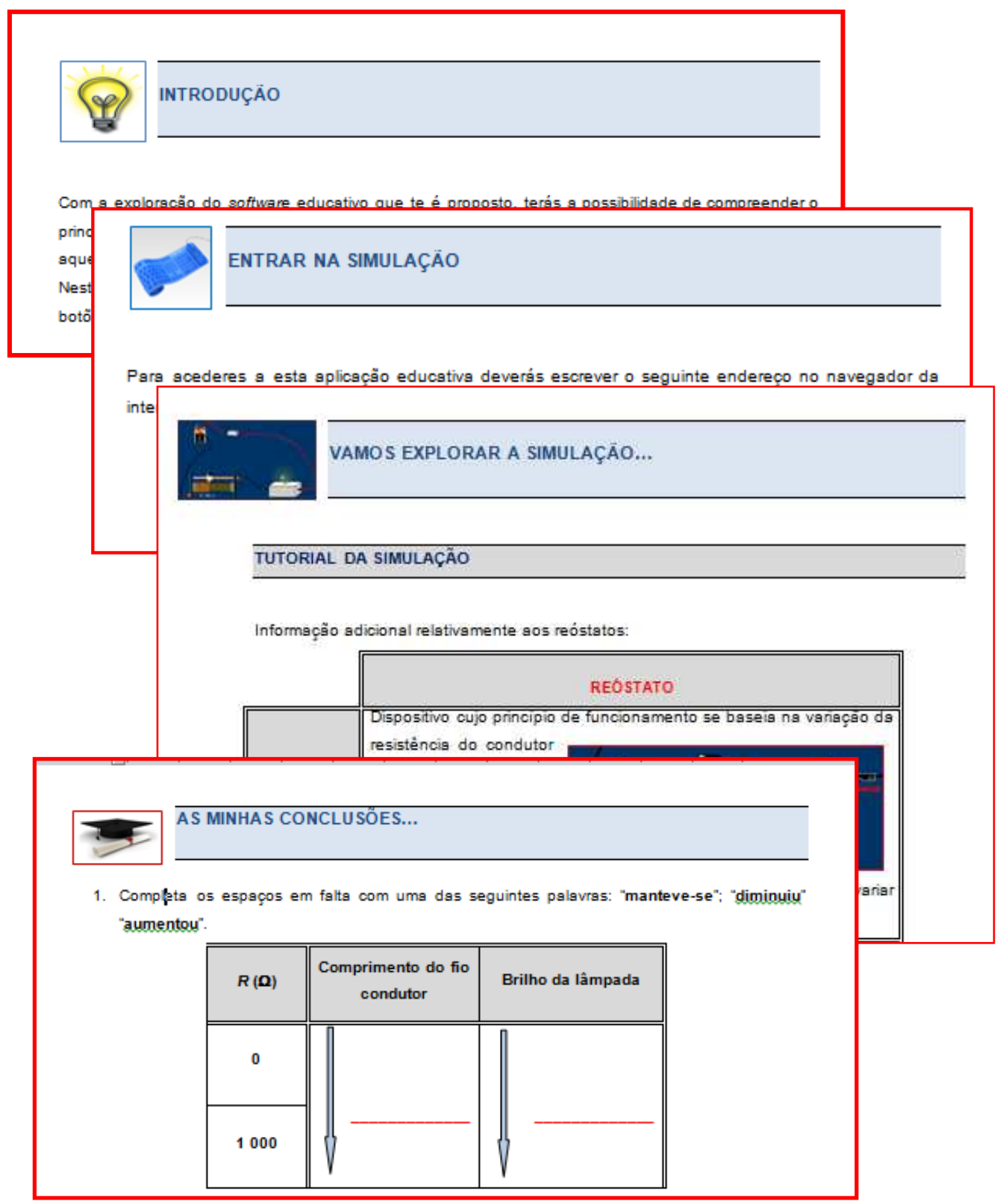

Caderno Brasileiro de Ensino de Física, v. 33, n. 3, p. 839-860, dez. 2016. 


\section{Reve - Curto-circuito}

REVE 10 - CURTO-CIRCUITO

$\left.\right|_{0} ^{0}$

INTRODUÇÄO

O vídeo educativo irá elucidar-te sobre o princípio físico subjacente ao curto-circuito.

De forma a poderes visualizar o vídeo, deverás aceder ao seguinte endereço da internet: http://www.youtube.comwatch?v=qOf350Gx-0l e sempre que necessário ef etua pequenas paragens, de forma a criares um espaço de discussão.

Neste Roteiro de Exploração de Vídeo Educativo (REVE) serás convidado(a) a visualizar o segmento de vídeo na totalidade e a responderes a um conjunto de questões.

\section{ENTRAR NO VÍDEO}

Para acederes a este vídeo educativo deverás escrever o seguinte endereçono navegador da internet:

http://www. voutube.com/watch?v=qOf350Gx-01

QUESTOES

Após a visualização do vídeo responde às questões que te serão apresentadas:

1. Como se caracteriza um fio ideal?

2. Indica na figura 1, por ação de setas, o caminho escolhido pela corrente elétrica. Fundamenta a tua resposta, recorrendo a linguagem científica.

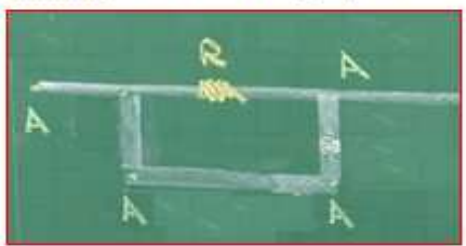

Figura 1 


\section{WebQuest}

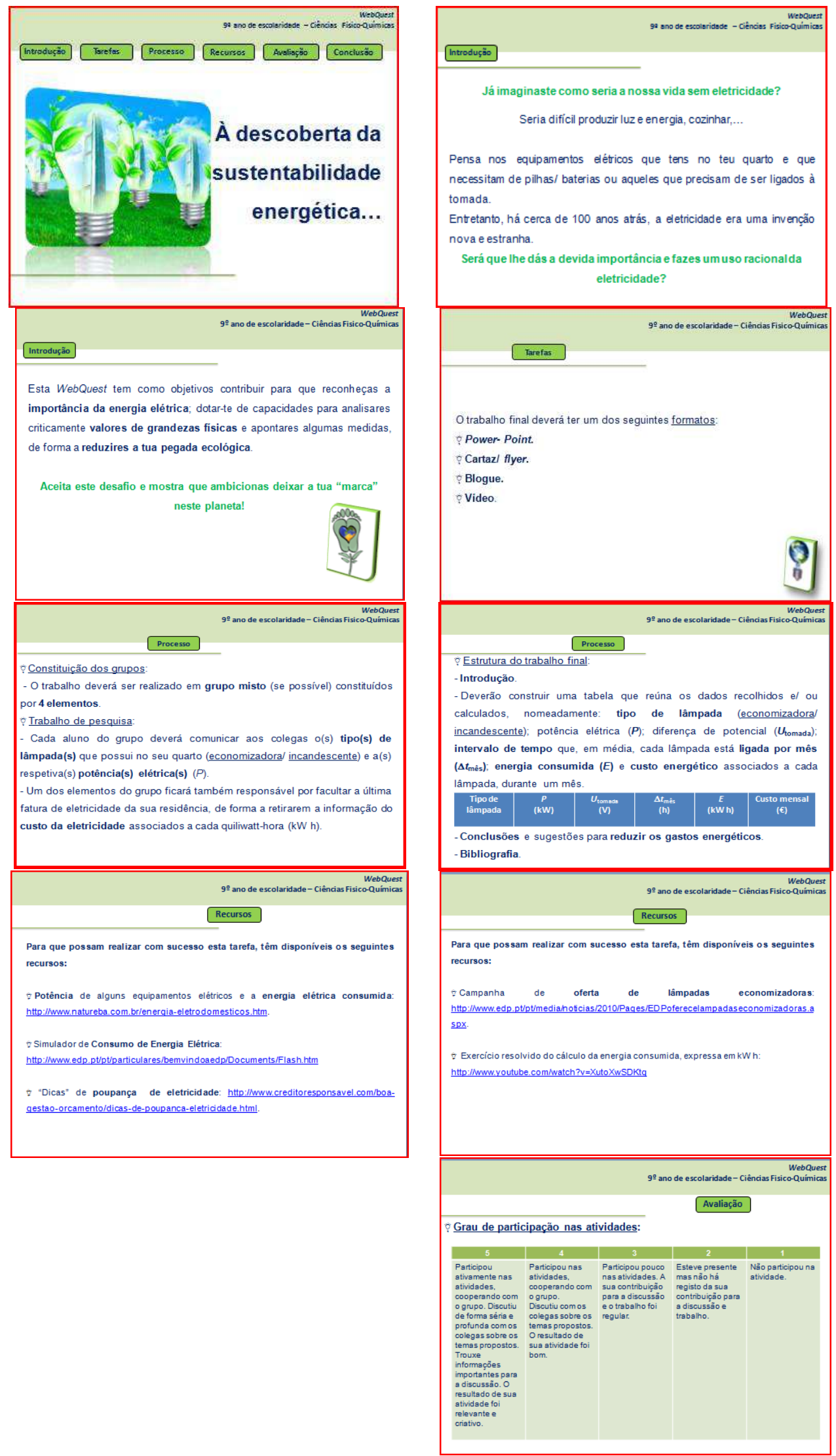




\section{Quiz}

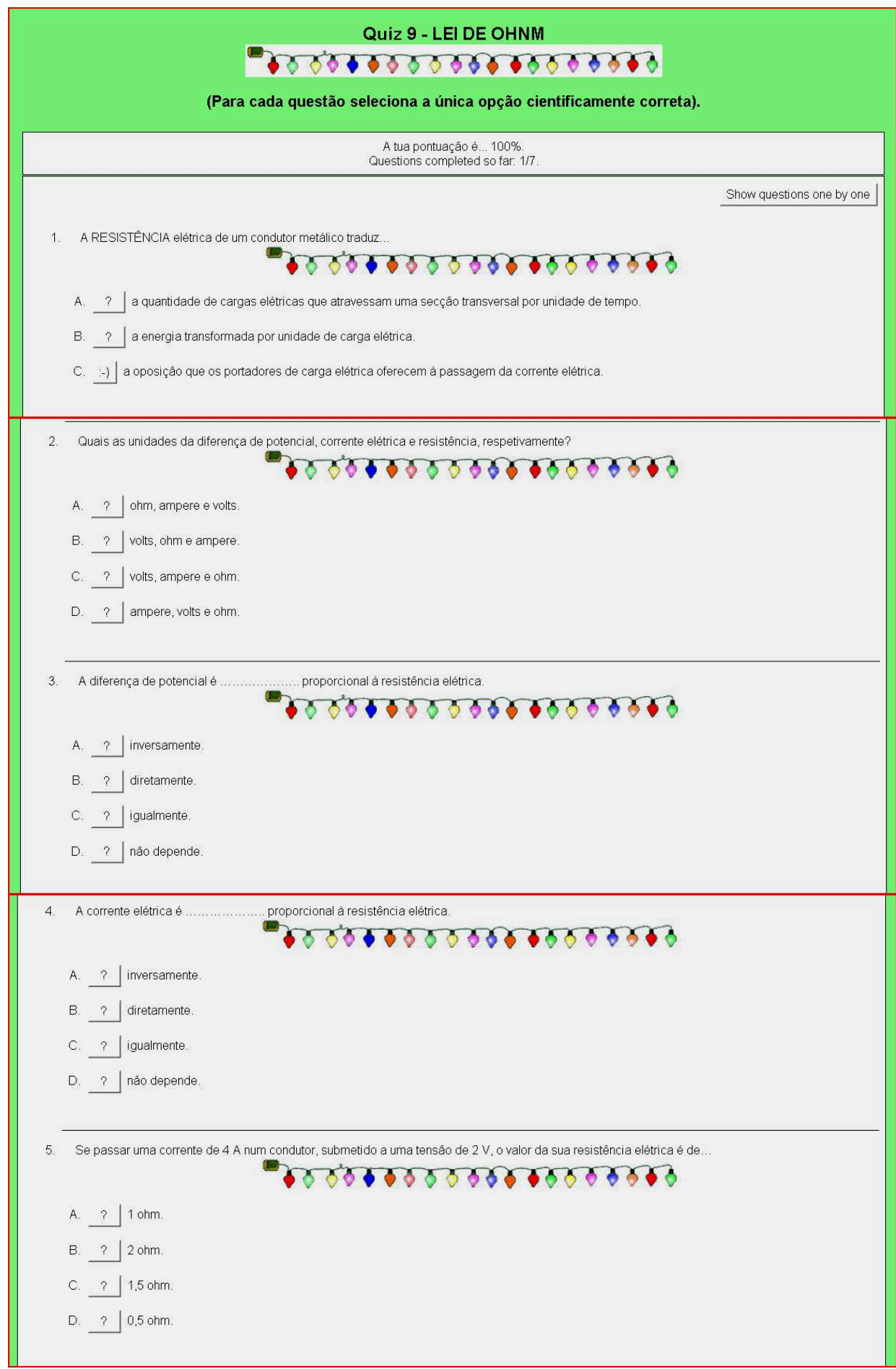




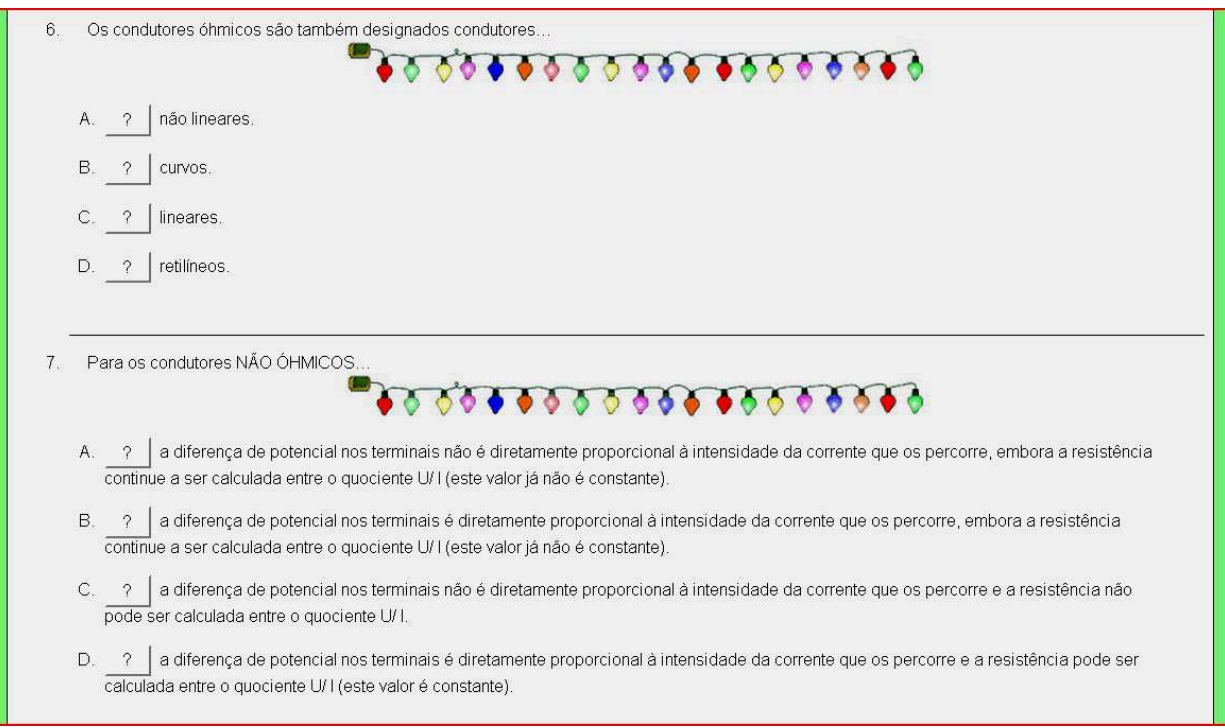

\section{Anexo B - Teste de conhecimentos}

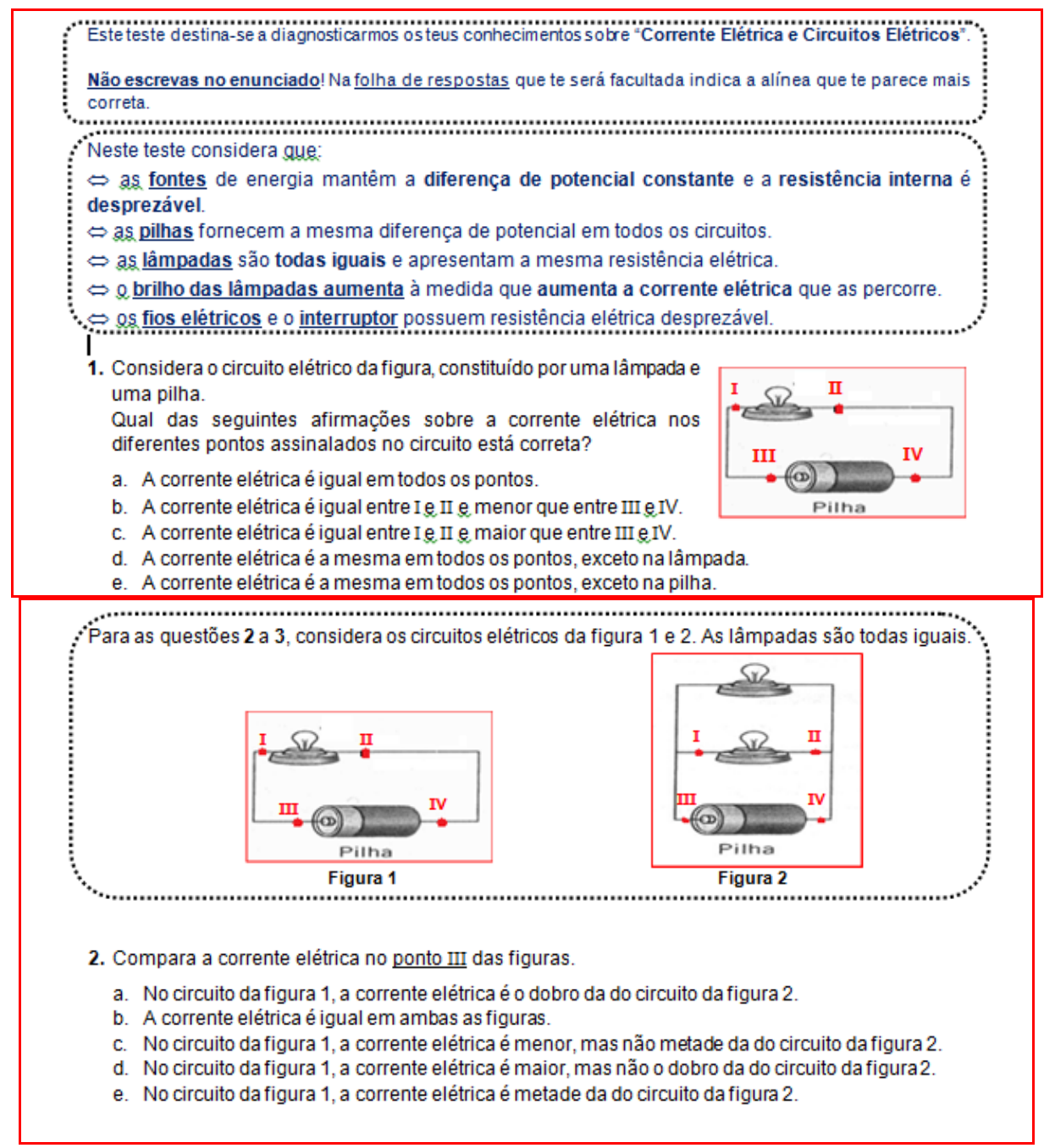


3. Compara a diferença de potencial entre os terminais I e II da lâmpada do circuito elétrico da figura 1 , com a diferença de potencial no caso do circuito da figura 2

a. No circuito da figura 1 , a diferença de potencial é o dobro da do circuito da figura 2

b. A diferença de potencial é igual em ambas as figuras.

c. No circuito da figura 1, a diferença de potencial é menor, mas não metade da do circuito da figura 2.

d. No circuito da figura 1, a diferença de potencial é maior, mas não o dobro da do circuito da figura 2 .

e. No circuito da figura 1, a diferença de potencial é metade da do circuito da figura 2

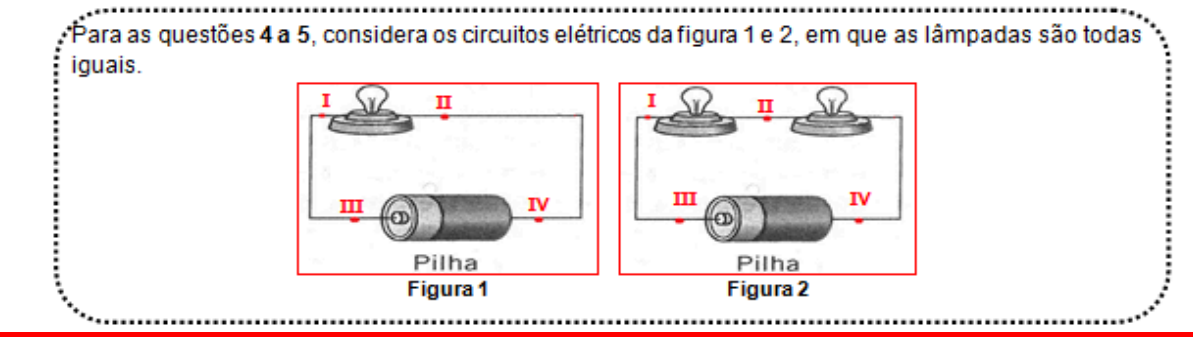

4. Compara a corrente elétrica no ponto III. das figuras

a. No circuito da figura 2, a corrente elétrica é o dobro da do circuito da figura 1.

b. A corrente elétrica é igual em ambas as figuras.

c. No circuito da figura 2, a corrente elétrica é menor, mas não metade da do circuito da figura 1 .

d. No circuito da figura 2, a corrente elétrica é maior, mas não o dobro da do circuito da figura 1.

e. No circuito da figura 2, a corrente elétrica é metade da do circuito da figura 1.

5. Compara a diferença de potencial entre os terminais I e II da lâmpada do circuito elétrico da figura 1 , com a diferença de potencial no caso do circuito da figura 2 .

a. No circuito da figura 2, a diferença de potencial é o dobro da do circuito da figura 1.

b. A diferença de potencial é igual emambas as figuras.

c. No circuito da figura 2, a diferença de potencial é menor, mas não metade da do circuito da figura 1.

d. No circuito da figura 2, a diferença de potencial é maior, mas não o dobro da do circuito da figura 1.

e. No circuito da figura 2, a diferença de potencial é metade da do circuito da figura 1.

Para as questões 6 a 7 , considera o circuito da figura seguinte, que traduz uma montagem constituída por duas lâmpadas, fios de ligação e uma pilha.

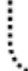

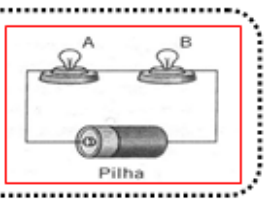

6. Seleciona, entre os materiais apresentados, o que poderia ser intercalado entre as lâmpadas A e B, de forma que estas continuem acesas.
a. Grafite (mina do lápis)
b. Vidro.
c. Madeira
d. Cortiça.

7. No circuito elétrico da figura, o brilho é..
a. maior na lâmpada $\mathrm{A}$.
b. maior nas proximidades do polo negativo da pilha
c. maior na lâmpada $B$
d. igual em ambas as lâmpadas.
e. Maior nas proximidades do polo positivo da pilha. 
8. Se ao circuito elétrico for adicionada mais uma pilha (ver figura ao lado), então o brilho da lâmpada A.

a. diminui.

b. aumenta.

c. não sofre qualquer alteração.

d. não há informação suficiente para tirarmos conclusões.

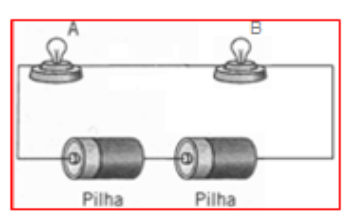

9. Para associarmos pilhas, tal como ilustradona figura,

a. O polo negativo (-) de uma pilha liga-se ao polo negativo $(-)$ da outra.

b. O polo positivo (+) de uma pilha liga-se ao polo positivo $(+)$ da outra.

c. O polo positivo (+) de uma pilha liga-se ao polo negativo (-) da outra

d. Ė indiferente a sequência de ligação de ambas as pilhas.

10. Considera os circuitos das figuras $1 \mathrm{e} 2$, em que as duas lâmpadas iguais encontram-se associadas de forma diferente.

Comparando a diferença de potencial nos terminais da lâmpada $\mathrm{A}$, de ambos os circuitos elétricos, na figura 1 esse valor é.

a. igual ao da figura 2 .

b. o dobro do da figura 2 .

c. metade do da figura 2

d. maior do que o da figura 2 .

e. Menor do que o da figura 2 .

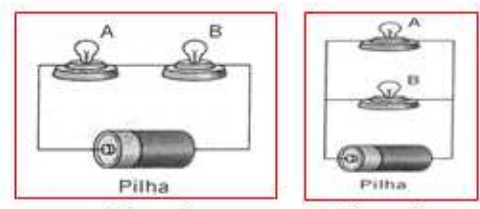

Figura 1

11. Considera o circuito elétrico ilustrado na figura, em que se colocou um interruptor (I) entre os terminais da lâmpada B

Ao fecharmos o interruptor.

a. aumenta o brilho da lâmpada $\mathrm{A}$

b. o brilho da lâmpada $\mathrm{A}$ permanece igual

c. diminui o brilho da lâmpada $\mathrm{A}$.

d. não possuímos informação suficiente para tirarmos conclusões.

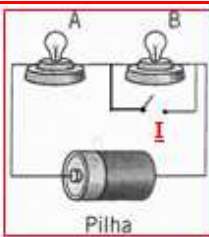

$\therefore$ Para as questões 12 a 14 , considera o circuito da figura seguinte, constituido por uma pilha, um interruptor fechado, dois instrumentos de medição (I e $\underline{\text { II }}$ ) e uma resistência elétrica.

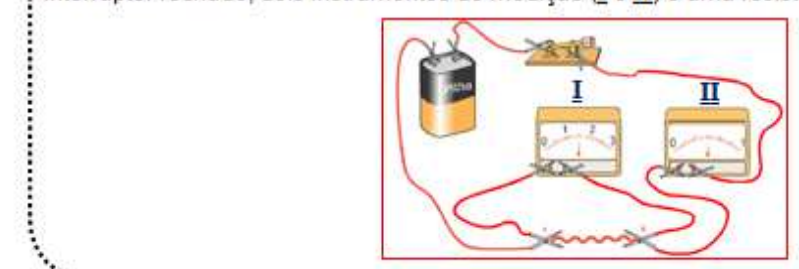

12. Os instrumentos de medição representados por $\underline{\mathbf{I}}$ e $\underline{\text { II }}$ são, respetivamente...
a. voltímetro e potenciómetro.
b. amperímetro e voltímetro.
c. ohmímetro e voltímetro.
d. amperímetro e potenciómetro.
e. voltímetro e amperímetro. 
13. A representação esquemática que traduz o circuito elétrico ilustrado na figura é

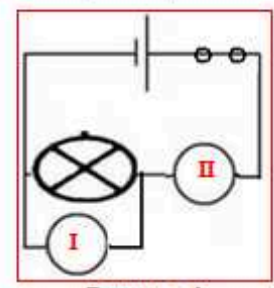

Esquema $\mathrm{A}$

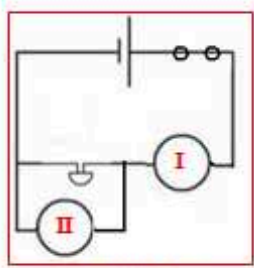

Esquema D

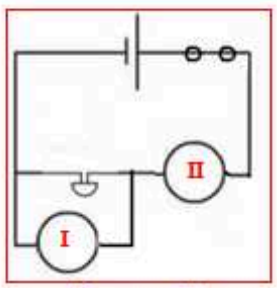

Esquema B

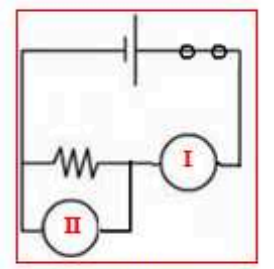

Esquema $\mathrm{E}$

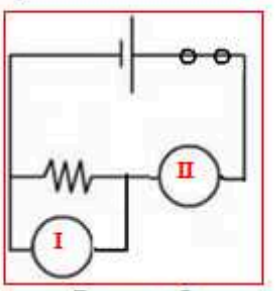

Esquema C

a. esquema $A$

b. Esquema B.

c. esquema $C$.

d. esquema $D$.

e. esquema $E$.

14. O gráfico que corresponde a um condutor óhmico é...
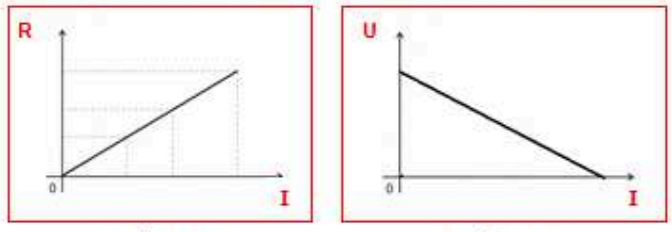

Gráfico B
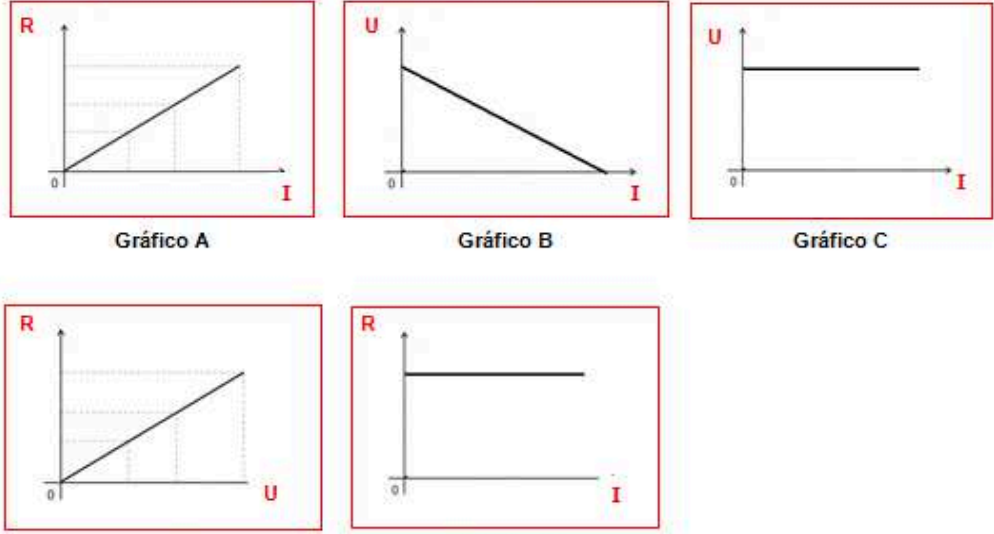

Gráfico D

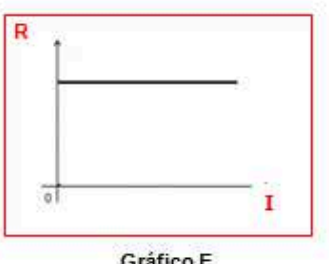

Gráfico $\mathrm{E}$

a. Gráfico $A$

b. Gráfico B.

c. Gráfico C.

d. Gráfico D.

e. Gráfico $\mathrm{E}$.

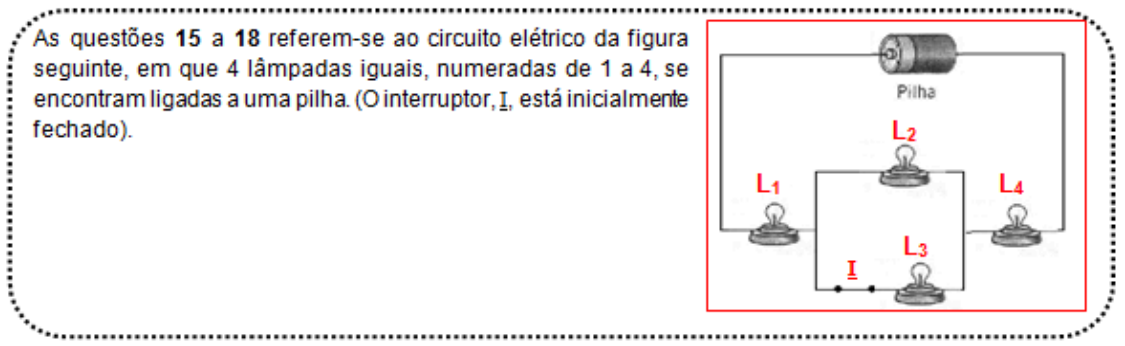


15. Qual das seguintes afirmações descreve corretamente o brilho nas lâmpadas?
a. $\mathrm{L}_{1}>\mathrm{L}_{2}=\mathrm{L}_{3}>\mathrm{L}_{4}$.
b. $L_{1}>L_{4}>L_{2}=L_{3}$
c. $L_{1}=L_{4}>L_{2}=L_{3}$.
d. $\mathrm{L}_{2}=\mathrm{L}_{3}>\mathrm{L}_{1}=\mathrm{L}$
e. Todas as lâmpadas apresentam o mesmo brilho.

16. Qual das seguintes afirmações descreve corretamente a diferença de potencial $(\boldsymbol{U})$ nas lâmpadas?
a. $U_{1}=U_{4}>U_{2}=U_{3}$
b. $U_{2}=U_{3}>U_{1}=U_{4}$
c. $U_{1}>U_{2}>U_{3}>U_{4}$
d. $U_{1}>U_{4}>U_{2}=U_{3}$
e. A diferença de potencial é igual em todas as lâmpadas

17. O que acontece ao brilho da lâmpada 1 , quando o interruptor I for aberto?
a. Aumenta.
b. Permanece igual.
c. Diminui.
d. Não há brilho
e. Não temos informação suficiente para responder.

18. O que acontece ao brilho da lâmpada 2 , quando o interruptor $\underline{\underline{I}}$ for aberto?

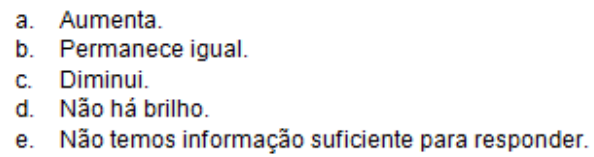

19. Em qual ou quais dos diagramas da figura, estão as duas resistências em série?
a. I.
b. II.
c. III.
d. I e III.
e. II e III.

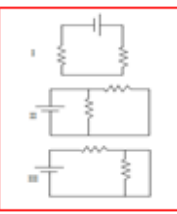

20. Considera o diagrama da figura que mostra um circuito elétrico com três resistores iguais, ou seja, $R_{\mathrm{A}}=R_{\mathrm{B}}=R_{\mathrm{C}}$. Qual a relação entre $\boldsymbol{I}_{\mathrm{A}}$ e $\boldsymbol{I}_{\mathrm{C}}$ ?
a. $I_{A}=1 / 3 I_{0}$
b. $I_{A}=1 / 2 \mathrm{I}$
c. $I_{\mathrm{A}}=I_{\mathrm{C}}$
d. $I_{\mathrm{A}}=2 I_{\mathrm{C}}$
e. $I_{\mathrm{A}}=3 I_{\mathrm{C}}$

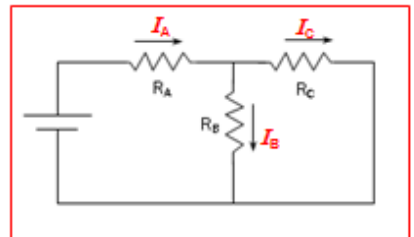

21. Observa a figura que traduz um circuito elétrico constituído por três pilhas, uma lâmpada e clipes. Se aumentarmos sucessivamente o número de clipes entre os dois terminais das pilhas, o brilho da lâmpada.
a. aumenta.
b. diminui.
c. mantém-se constante.
d. nada se pode afirmar.

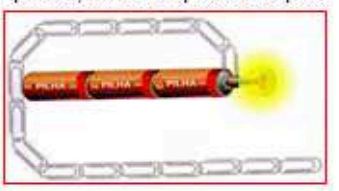

22. No circuito elétrico da figura, os componentes 1, 2, 3 e 4, traduzem, respetivamente, os seguintes efeitos da passagem da corrente elétrica...

a. térmico, magnético, térmico e químico.

b. químico, magnético, térmico, térmico.

c. térmico, químico, magnético, térmico.

d. químico, térmico, magnético, térmico.

e. nenhuma das hipóteses anteriores.

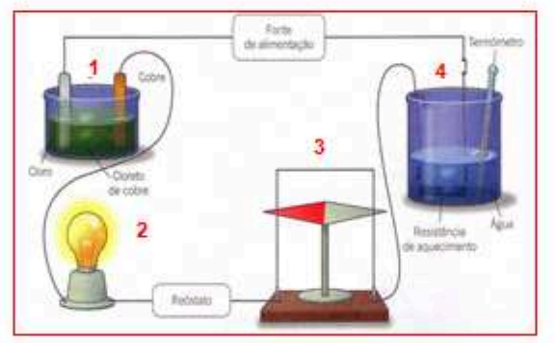


23. As consequências de um choque elétrico estão relacionadas com..

a. apenas a corrente elétrica que atravessa o corpo da pessoa.

b. a diferença de potencial, bem como o caminho que atravessa o corpo da pessoa.

c. a corrente elétrica, bem como pelo caminho que atravessa o corpo da pessoa.

d. a resistência elevada do corpo da pessoa.

e. a diferença de potencial a que é sujeito o corpo da pessoa. 